\title{
Temporal suppression of long-latency click-evoked otoacoustic emissions
}

\author{
Verhulst, Sarah; Harte, James; Dau, Torsten
}

Published in:

Proceedings of the 29th annual international conference of the IEEE engineering in Medicine and Biology Society, 2007

Link to article, DOI:

10.1109/IEMBS.2007.4352695

Publication date:

2007

Document Version

Publisher's PDF, also known as Version of record

Link back to DTU Orbit

Citation (APA):

Verhulst, S., Harte, J., \& Dau, T. (2007). Temporal suppression of long-latency click-evoked otoacoustic emissions. In Proceedings of the 29th annual international conference of the IEEE engineering in Medicine and Biology Society, 2007: Sciences and Technologies for Health (pp. 1932-1936). IEEE.

https://doi.org/10.1109/IEMBS.2007.4352695

\section{General rights}

Copyright and moral rights for the publications made accessible in the public portal are retained by the authors and/or other copyright owners and it is a condition of accessing publications that users recognise and abide by the legal requirements associated with these rights.

- Users may download and print one copy of any publication from the public portal for the purpose of private study or research.

- You may not further distribute the material or use it for any profit-making activity or commercial gain

- You may freely distribute the URL identifying the publication in the public portal 


\title{
Temporal suppression of long-latency click-evoked otoacoustic emissions
}

\author{
Sarah Verhulst, James M. Harte, Torsten Dau
}

\begin{abstract}
A comprehensive set of results from double click suppression experiments on otoacoustic emissions (OAEs) have been presented by Hine and Thornton [1] and Kapadia and Lutman [2]. They found that suppression of a click-evoked otoacoustic emission (CEOAE) varied with the timing and level of a suppressor-click presented close in time to the testclick. Maximal suppression was found when the suppressorclick led the test-click by 2-4 ms. The double click suppression experiment set out by Hine and Thornton [1] was repeated here and the analysis extended to the 'long-latency' CEOAE (duration $>20 \mathrm{~ms}$ ) whereas previous studies only focused on the 'short-latency' CEOAE (duration $<20 \mathrm{~ms}$ ). The hypothesis was that suppression would continue over the long-latency CEOAE since this region is probably dominated by spontaneous OAEs (SOAEs) synchronising with the click stimulus. The results for two exemplary subjects showed that the nonlinear suppression effect remained on the long-latency CEOAE, indicating that both SOAEs and CEOAEs originate from the same cochlear nonlinearities, as earlier suggested by Kemp and Chum [3]. The apparent similar origin of both types of emissions implies that the same temporal effects influence their responses.
\end{abstract}

\section{INTRODUCTION}

The human auditory system is known to contain various nonlinearities; one source is commonly believed to be a feedback loop within the cochlea, often described as the 'active process'. This was first postulated by Gold [4], whose theory stated that to account for the high frequency selectivity, the filters in the cochlea must be active. This mechanism sharpens the response of the auditory filters and it compresses the dynamic range of the cochlear response as well. The active process is also believed to give rise to otoacoustic emissions (OAEs), first demonstrated by Kemp [5]. The vast majority of normally hearing adults $(98 \%)$ have measurable transient- or click-evoked otoacoustic emissions (TEOAEs or CEOAEs, Probst et al. [6]). When click stimuli are used, the majority of ears show emission spectra containing several discrete frequencies, which are known as dominant frequencies. These dominant frequencies may be spontaneous otoacoustic emissions (SOAEs), synchronized by the transient stimuli so that they become 'long-latency' TEOAEs (duration > $20 \mathrm{~ms}$ ) [6]. Alternatively they may also be generated by oscillations at specific frequencies that are only present when evoked, but relatively weakly damped [6]. SOAEs are often thought of as a consequence of particular locations on the cochlea having active mechanisms in a region of instability [7]. The nonlinearity in otoacoustic

This work was supported by the Technical University of Denmark

S. Verhulst, J.M. Harte and T. Dau are with the Centre for applied hearing research (CAHR), Ørsted-DTU, Technical University of Denmark, Ørsteds plads bldg.352, DK-2800 Kgs. Lyngby.

Corresponding author:sv@oersted.dtu.dk emissions can be measured in several ways, including the suppression of one emission by the presentation of a suppressor-stimulus, [1][2][3][8]. The degree of suppression varies systematically with the timing and the level of the suppressor-click [1][2], being greatest for suppressor-clicks occurring 2-4 ms before the test-click, particularly at lower levels of the suppressor. Almost no suppression occurred when the suppressor-click led the test click by more than 6 ms, which was set by Hine and Thornton [1] as the temporal nonlinearity limit of the cochlea. Some modeling attempts have been partially successful in explaining the suppression data in terms of the nonlinear compressive growth curve for OAEs [9][10]. Both the studies of Kapadia and Lutman [2] and Hine and Thornton [1] focused on suppression of small time frames (3-5 ms) of the short-latency CEOAE (duration $<20 \mathrm{~ms}$ ). The aim of the present study was to investigate suppression of the long-latency CEOAE response, as this region might be dominated by SOAEs. The hypothesis was that suppression would continue on the long-latency CEOAE if SOAEs and CEOAEs originate from identical cochlear nonlinearities inside the cochlea, as suggested by Kemp and Chum [3]. The double click suppression experiment set out by Hine and Thornton [1] was repeated, extended and the analysis was carried out on data from individual subjects.

\section{MATERIALS AND METHODS}

\section{A. Recording CEOAEs}

The stimuli used in the experiment were generated using Matlab and sent to an ADI-8Pro, a Hi-Precision 24 bit A/D-D/A converter, through a sound card operated by the SoundMex ${ }^{1}$ v2.15 set of dlls. After the D/A conversion, the signal was passed through a DT PA5 programmable attenuator and a DT HB7 headphone driver. The attenuated signal was presented to the test subject via an ER-2 probe. Foam eartips were used to ensure a tight seal in the ear canal. The click response was recorded with an ER-10dB low noise microphone and the preamplified signal was passed through a Krohn-Hite analog bandpass filter with cut-off frequencies of $600 \mathrm{~Hz}$ and $5000 \mathrm{~Hz}$ for noise rejection purposes. After filtering, the recorded signal was sent back through the A/D converter and stored digitally. The test subjects were lying down in a soundproof booth with the measurement probe in the left ear canal for all recordings. An appropriate choice of eartip was sought and any wax removed from the ear canal prior to probe insertation. The subjects were asked to keep head movements to a minimum

\footnotetext{
${ }^{1}$ Copyright HörTech gGmbH, Oldenburg Germany
} 
and to avoid touching the face during the measurement. Test subjects were screened to ensure all had a hearing loss of less than $15 \mathrm{~dB}$ across the audiogram.

\section{B. Experimental paradigm}

Click stimuli were used in order to activate a broad range of frequency regions on the basilar membrane and to measure a spectrally rich CEOAE recording. The levels of the stimuli were chosen as $65 \mathrm{~dB}$ peak equivalent sound pressure level (peSPL) to obtain a response in the nonlinear compressive region of the growth curve for CEOAEs [6]. The inter-click intervals investigated ranged from 0.2 to 8 $\mathrm{ms}$. The exact ICI values were chosen to be identical to those used in the study by Hine and Thornton in 2002, except two extra values for longer ICIs were added. ICI $=$ $\left[\begin{array}{llllllllll}0.2 & 0.33 & 0.5 & 1 & 2 & 3.33 & 5 & 6 & 7 & 8\end{array}\right] \mathrm{ms}$. The ICIs of 7 and 8 $\mathrm{ms}$ were added in order to test the temporal nonlinearity memory limit of $6 \mathrm{~ms}$, set by Hine and Thornton [1]. The analysis window for the CEOAE response recording was $39 \mathrm{~ms}$ long (i.e. double the size of the recording window in Hine and Thornton's experiment) to investigate the longer latency click response associated with synchronised SOAEs. Hine and Thornton's experiment focussed only on test subjects without SOAEs and on the short-latency click response, hence their shorter analysis window. For each ICI, two responses were measured: the double-click response and the single-click response with the click at the position of the second click of the double-click stimulus. Subtraction of the single-click response from the double-click response led to the derived suppressed response, in which the influence of the ICI onto the response could be investigated. The single-click response was measured again for every ICI value to reduce the probe fitting error induced by movement of the test subject. For every measurement point, a minimum of 2000 click recordings were made in order to get to an averaged click response with a reasonable signal-to-noise ratio. The measurement was split up into three runs of about 30 minutes in order to make the measurement more comfortable for the test subjects. This is justified because the amplitudes and frequencies of the OAE components do not vary substantially over time [6]. All runs were conducted on the same day to rule out middle-ear pressure changes that do have an influence on the amplitude of the OAE recordings. Repeatability could be an issue when splitting up a measurement into different runs but was investigated and found to be good [11]. Five subjects were tested in this experiment and results from two exemplary subjects with long-latency CEOAEs are discussed here.

\section{Post Processing}

The post processing was carried out offline with the aid of Matlab and an artifact rejection algorithm was included. The measured click responses were scaled to obtain the amplitudes in Pascals $(\mathrm{Pa})$ to make a comparison across subjects possible. This was done by first scaling the recorded values to

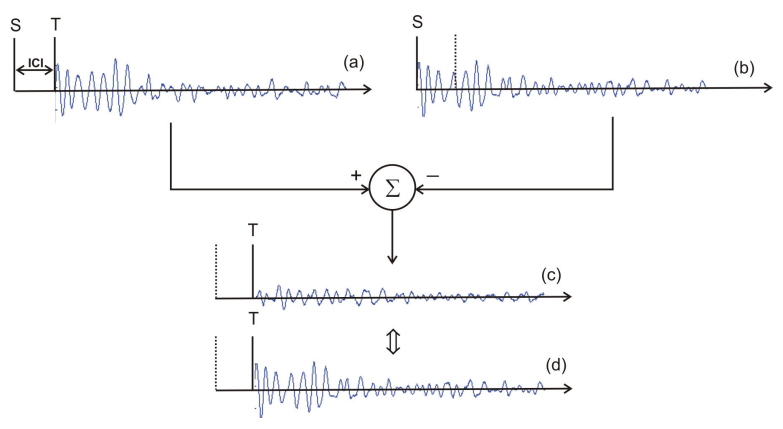

Fig. 1. Trace (a) shows the CEOAE response after double-click stimulation. The suppressor-click $(\mathrm{S})$ was presented at a certain ICI before the testclick (T). Trace (b) shows the CEOAE response to a single click. The click of trace (b) was aligned with the suppressor-click of trace (a) before subtraction. Trace (c) shows the derived suppressed response after subtraction of trace (b) from trace (a) and the subtraction was done to delete the influence of the first click and maintain the influence of the ICI. Trace (d) shows the CEOAE response to a single click where the click was aligned with the test-click of the derived suppressed response and is referred to as the unsuppressed response. Suppression was then found as the difference between the unsuppressed CEOAE (d) and the derived suppressed CEOAE (c), calculated in $L_{r m s}$ as in (1). Positive suppression was found when the amplitude of the derived suppressed CEOAE was smaller than the amplitude of the unsuppressed response.

the voltage dynamic range of the A/D converter. Afterwards, a compensation for the pre-amplifier was included and the obtained voltages were scaled to the microphone sensitivity, which was $50 \mathrm{mV} / \mathrm{Pa}$ for the ER-10dB microphone for all considered frequencies, leading to response amplitudes in Pascals. The overall click response, measured from each subject, contains an early-latency linear response, associated with the ear-canal and middle-ear transfer function. Data analysis was carried out on the CEOAE part of the click responses, which is the nonlinear component associated with the cochlea. A separation technique was developed to determine the time point from where the click response was considered to be nonlinear. This time point was set on $\tau_{s}=9 \mathrm{~ms}$ after the last click onset; details on this technique can be found in [11]. The response to a single-click stimulus was referred to as the unsuppressed response and was aligned with the first click of the double-click stimulus case. The single-click response was then subtracted from the double-click response to remove the component due to the first click from the suppressed response. Suppression in CEOAEs was measured as the difference between the derived suppressed response and the unsuppressed response, as shown in Fig. 1. Suppression occurs because CEOAEs are inherently nonlinear; for linear signals, linear superposition would be applicable and the suppressed response would be identical to the unsuppressed result. Suppression levels were calculated as the difference between the rms level in $\mathrm{dB}$ SPL of the unsuppressed CEOAE and the rms level in $\mathrm{dB}$ SPL of the derived suppressed CEOAE. The rms levels were calculated for time frames of $3 \mathrm{~ms}$ starting from $\tau_{s}=9 \mathrm{~ms}$ after the last click onset (for the unsuppressed- and derived suppressed CEOAE) by use of (1) for $N=3 \mathrm{~ms} \cdot f_{s}$, where $N$ stands for the length in samples of a 3-ms long analysis 
window.

$$
L_{r m s}\left(t_{p}\right)=20 \log _{10}\left[\frac{1}{N-1} \sum_{i=1}^{N}\left(x_{i}-\mu\right)^{2}\right]^{1 / 2}
$$

A value for $L_{r m s}$ is found for every 3-ms long time frame of the CEOAE with $t_{p}$ defining the time frame. $x_{i}$ are the samples of the CEOAE and $\mu$ stands for the mean of the CEOAE within the considered $3 \mathrm{~ms}$ window.

\section{RESULTS}

\section{A. Unsuppressed CEOAEs}

The unsuppressed CEOAE was obtained from single-click recordings. The rms levels of the CEOAEs were calculated according to (1) for $3 \mathrm{~ms}$ windows of the response and levels for two exemplary subjects are plotted in Fig. 2. The rms levels for test subject 1 (left panel) can be split up into two regions. The first region comprised of the time frames from $9 \mathrm{~ms}$ up to $21 \mathrm{~ms}$, with values reported between 7 and $14 \mathrm{~dB}$ SPL. The second region ranged from 21 to $39 \mathrm{~ms}$ with levels ranging from -4 to $7 \mathrm{~dB}$ SPL. This overall level reduction over all considered time frames can be explained by the CEOAE time series for this particular subject. The top panel of Fig. 3 shows the unsuppressed CEOAE time series for this subject, where a strong CEOAE response is seen from 9 to $21 \mathrm{~ms}$, with the amplitude decreasing for the long-latency region. The amplitudes of SOAE components are typically much lower than the short-latency CEOAE components [6], hence the amplitude reduction of the longer latency CEOAE. The rms levels on Fig. 2 do not vary dramatically over ICI. This was expected since Fig. 2 is simply a repetition of the singe-click CEOAE, recorded at different time instants during the measurement. They were plotted here per ICI to present the unsuppressed CEOAE that was compared later on to the suppressed CEOAE for a certain ICI to form suppression. The repetition of the single-click measurement was done to ensure minimal impact of probe movement that could introduce an elevated noise floor, therefore Fig. 2 represents the degree of repeatability for the single-click results. The rms levels for test subject 2 in the right panel of Fig. 2 follow the same tendency as the data for test subject 1 in the left panel. Very small level differences might be observed when comparing the data of subject 1 to subject 2 and can be related to the amplitude variation between subjects for recording CEOAEs, as shown on the lower curve on Fig. 3. Amplitude variation in CEOAE recordings depends on the number and frequencies of dominant SOAEs and the properties of the middle-ear for a particular subject [6].

\section{B. Derived Suppressed CEOAEs}

The rms levels of the derived suppressed CEOAE are plotted in Fig. 4 for the two test subjects. In contrast to the rms levels of the unsuppressed CEOAEs (Fig. 2), the levels did vary as a function of ICI. The rms levels of both test subjects ranged between 2 and $12 \mathrm{~dB}$ SPL for all

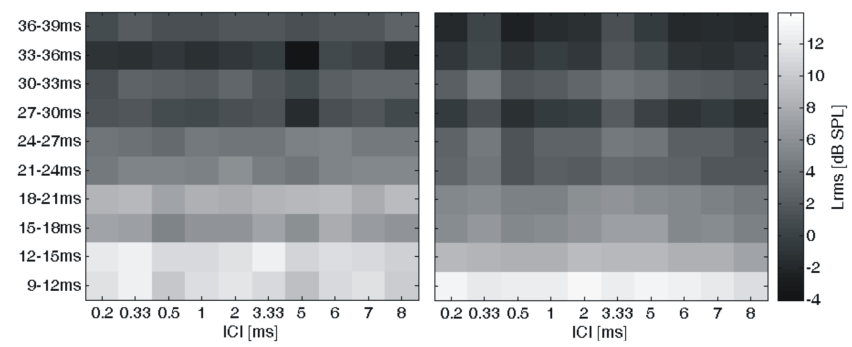

Fig. 2. Root-mean square levels $\left(L_{r m s}\right)$ of the unsuppressed response per ICI, analysed in 3-ms long time frames for two different test subjects. The unsuppressed CEOAEs were plotted per ICI value even though they represent single-click responses that should be nearly identical as they are not a function of ICI. To obtain a value for suppression, a single-click response was measured right after a double-click response, so the CEOAEs plotted stand for the click response that were compared later on with the derived suppressed CEOAE for a particular ICI.

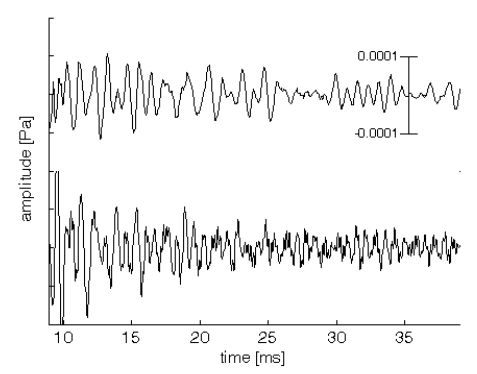

Fig. 3. Unsuppressed CEOAE for two test subjects plotted from $9 \mathrm{~ms}$ after the click onset.

time frames and ICIs, except for ICIs between 0.5 and 5 ms where an overall level reduction of $4 \mathrm{~dB}$ was observed for subject 1 (left panel). Subject 2 (right panel) shows an overall level reduction of $5 \mathrm{~dB}$ for ICIs of 0.5 to $2 \mathrm{~ms}$. One can qualitatively compare the rms levels of the derived suppressed responses of the five measured subjects to the data from Hine and Thornton [1]. The top panel of Fig. 5 shows a reproduction of Hine and Thornton's mean data of the derived suppressed response over seven subjects per ICI [1]. Three time frames are shown in the short-latency region of the CEOAE. The bottom panel of Fig. 5 shows the obtained mean data over five subjects plotted in the same
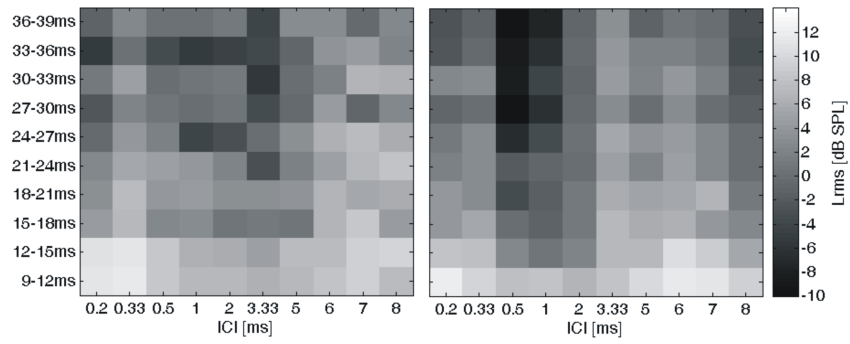

Fig. 4. Root-mean square levels $\left(L_{r m s}\right)$ of the suppressed response per ICI, analysed in 3 -ms long time frames for two different test subjects. 

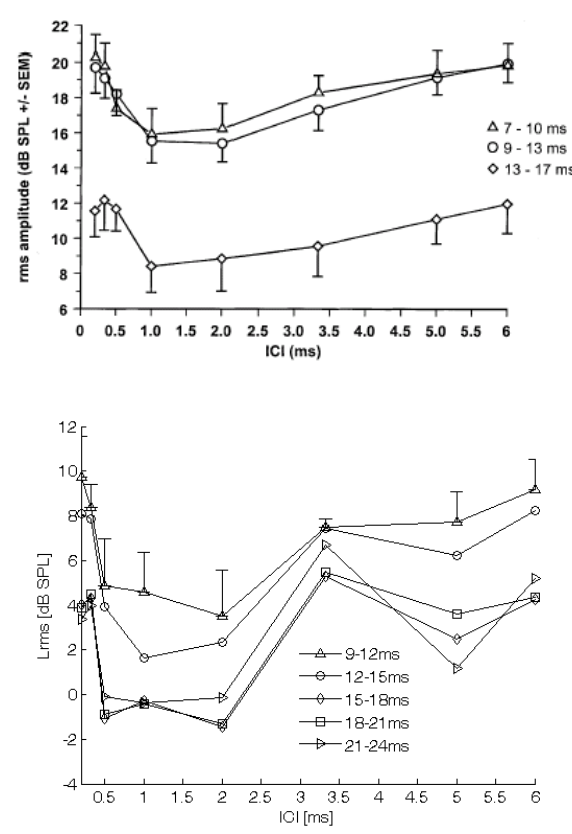

Fig. 5. Top panel: rms levels of the suppressed click response versus ICI plotted for different time frames after the last click onset [Taken from Hine \& Thornton (2002)]. Bottom panel: rms levels $\left(L_{r m s}\right)$ of the derived suppressed click response versus ICI, plotted for different time frames after the last click onset as an average over five test subjects. The standard error across subjects was plotted on the rms levels of the 9-12 ms time frame and representative for all the time frames considered. Note the different scales between the top and bottom panel.

way, except that the first five time frames of the CEOAE are shown. It was observed that the data of Hine and Thornton [1] is on average $10 \mathrm{~dB}$ higher than the reported results for the derived suppressed response. This offset might be due to differences in calibration methods, but little is known about the procedure that Hine and Thornton [1] used and therefore the comparison between the data is conducted qualitatively in the following ${ }^{2}$. The mean data of the five subjects resembled the data of Hine and Thornton (top panel of Fig. 5) reasonably well. A dip in the levels of the derived suppressed CEOAE by about $5 \mathrm{~dB}$ was reported for ICI intervals of $0.5,1$ and $2 \mathrm{~ms}$, regardless of the considered time frame (bottom panel of Fig. 5), and this was in agreement with the maximal dip found by Hine and Thornton [1]. For the rms levels on the top panel of Fig. 5, a level reduction around $8 \mathrm{~dB}$ was observed from the first two time frames onto the third, 13 to $17 \mathrm{~ms}$ time frame. The average data on the bottom panel of Fig. 5 showed a reduction of about $3 \mathrm{~dB}$ between the $12-15 \mathrm{~ms}$ and the 15-18 ms time frame. The reason for this smaller level reduction could be explained by the nature of the CEOAEs for the subjects used. All subjects had SOAEs that rang up to $39 \mathrm{~ms}$ after click onset and this implied that the response

\footnotetext{
${ }^{2} \mathrm{~A}$ direct and quantitative comparison between the literature data and the measured results is possible when talking about suppression in the next section. The suppression parameter is independent of the calibration method as it describes the difference between absolute levels of the responses.
}

did not decay down to the noise floor after the short latency region as was the case for the subjects of Hine and Thornton.

\section{Suppression}

A more reliable value for the level reduction, caused by a click represented with a certain ICI before a click response, was found by looking at suppression. This was defined as the level difference between the unsuppressed CEOAE and the derived suppressed CEOAE. The suppression parameter was invariant to absolute level changes over subjects due to the strength of their emissions, which made a quantitative comparison across subjects possible. The rms levels of suppression for $3 \mathrm{~ms}$ time frames after the last click onset are shown for the two subjects (Fig. 6) as a function of ICI. Both subjects show suppression between ICIs of 0.5 and $5 \mathrm{~ms}$; outside of these boundaries both subjects showed level reductions between -4 and $4 \mathrm{~dB}$. Subject 1 (left panel of Fig. 6) showed level reductions between 4 and $10 \mathrm{~dB}$ for ICIs of 1,2 and $3.33 \mathrm{~ms}$ with maximal suppression reported for when the ICI was $3.33 \mathrm{~ms}$. Subject 2 (right panel of Fig. 6), showed high suppression levels for ICIs of $0.5,1$ and $2 \mathrm{~ms}$ with maximal suppression observed for an ICI of $2 \mathrm{~ms}$. For both subjects, suppression disappears as the inter-click interval reaches $6 \mathrm{~ms}$. The data for both subjects follows the literature data quite well. For a stimulus level of $65 \mathrm{~dB}$ peSPL, Hine and Thornton [1] found that the maximal dip in the suppressed response $(5 \mathrm{~dB})$ was observed for a 1-2 ms inter-click interval [1]. Kapadia and Lutman also reported maximal suppression with a value around $5 \mathrm{~dB}$ when the suppressor click led the test click by $2 \mathrm{~ms}$, for a click stimulus level of $60 \mathrm{~dB}$ peSPL [2]. Maximal suppression for most subjects was found for ICIs of $0.5 \mathrm{~ms}$ and $1 \mathrm{~ms}$, with values ranging from 3 to $5 \mathrm{~dB}$, as can be verified from Fig. 7. The suppression values per ICI for all subjects were consistent over the time frames considered. All subjects had SOAEs synchronizing with the click stimuli causing the CEOAE to ring until the end of the measurement window of $39 \mathrm{~ms}$. The longer latency response is associated with the SOAEs and observing suppression over the whole time range of the recording supports the hypothesis that both the CEOAE and the SOAE originate from similar mechanisms inside the cochlea.

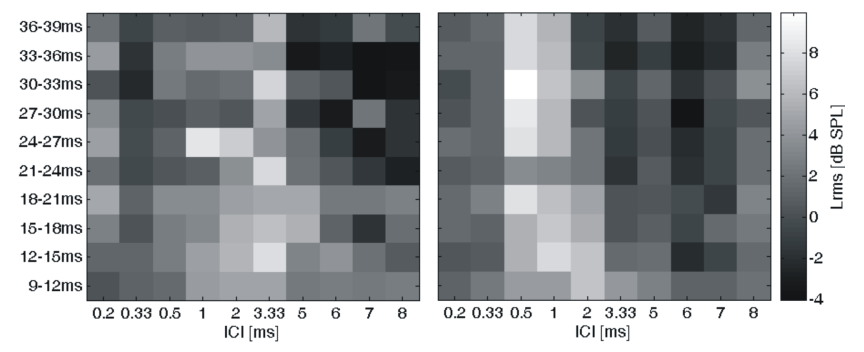

Fig. 6. Root-mean square levels $\left(L_{r m s}\right)$ of the suppression per ICI, analysed in 3-ms long time frames for two different test subjects. 


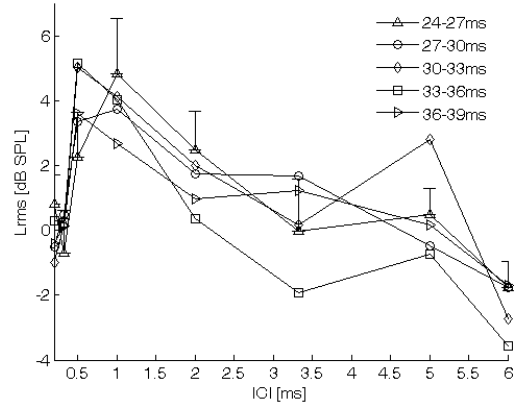

Fig. 7. rms levels $\left(L_{r m s}\right)$ of suppression versus ICI, plotted for different long-latency time frames after the last click onset as an average over five test subjects. The standard error across subjects was plotted on the rms levels of the $24-27 \mathrm{~ms}$ time frame and representative for all the time frames considered.

\section{DISCUSSION AND FUTURE WORKS}

Cochlear nonlinearities were investigated by the use of CEOAE suppression recordings, when a suppressor click was presented before a test click, with a certain inter-click interval spacing them. The double-click experiment proposed by Hine and Thornton [1] and Kapadia and Lutman [2] was repeated and extended to relate the influence of an inter-click interval causing suppression to cochlear nonlinearities. In particular, the presence of SOAEs was investigated in the CEOAE recordings. The single-click CEOAE response, or the unsuppressed CEOAE response, gave an indication of the duration and strength of a subjects CEOAEs. The derived suppressed CEOAE response, used to determine the influence of a certain inter-click interval on the amplitude of a CEOAE, showed suppression in a region for ICIs between 1 and $5 \mathrm{~ms}$ for all subjects. The experimental data for the short-latency region ( 9 to $20 \mathrm{~ms}$ after click onset) of the derived suppressed CEOAE response were qualitatively comparable to the experimental data of Hine and Thornton [1]. A separation into a subject group with long CEOAEs and one with short CEOAEs is recommended for future studies and might give a closer resemblance to the literature data. Suppression was reported for the same ICI values as in previous experiments [1][2] and the $6 \mathrm{~ms}$ limit for the temporal nonlinearity memory of the cochlea was confirmed since very little suppression was reported for ICIs longer than $6 \mathrm{~ms}$. In fact, at an ICI of $6 \mathrm{~ms}$, augmentation of the responses is observed for the longer-latency regions with a value around $3 \mathrm{~dB}$. Augmentation was reported before for long-latency CEOAEs by Tavartkiladze et al. [8] for ICIs of 6 and $7 \mathrm{~ms}$. It was interesting to observe augmentation of the long-latency CEOAE, but this needs needs further investigation. The suppression reported for certain ICIs is believed to come from temporal effects inside the cochlea, influencing the amplitude of a CEOAE because its values cannot solely be explained by the nonlinear compressive input/output curve for CEOAEs. The experimental results showed that the $5 \mathrm{~dB}$ suppression value was still present in the long-latency region of the response, i.e. after $20 \mathrm{~ms}$ of the last click onset. Since this region is probably dominated by SOAE components synchronizing with the CEOAE it was indicated that both SOAEs and CEOAEs originate from the same cochlear nonlinearities inside the cochlea, as was suggested by Kemp and Chum [3]. The apparent similar origin of both types of emissions implies that the same temporal effects influence their responses.

\section{ACKNOWLEDGMENTS}

The software for measuring OAEs was designed in cooperation with the Carl von Ossietzky Universität in Oldenburg.

\section{REFERENCES}

[1] J. E. Hine and A. R. D. Thornton, "Temporal nonlinearity revealed by transient evoked otoacoustic emissions recorded to trains of multiple clicks", Hearing Research, vol. 165, 2002, pp 128-141.

[2] S. Kapadia and M. E. Lutman,"Nonlinear temporal interactions in click-evoked otoacoustic emissions. II. Experimental data", Hearing Research, vol. 146 nr.1-2, 2000, pp 101-120

[3] D. T. Kemp and R. A. Chum, "Properties of the generator of stimulated otoacoustic emissions", Hearing Research, vol. 2, 1980, pp 213-232.

[4] T. Gold, "Hearing. II. The physical basis of the action of the cochlea", Proceedings of the Royal Society of London, vol. 135 nr. 881, 1948, pp 492-498

[5] D. T. Kemp, "Stimulated acoustic emissions from within the human auditory system", J Acoust Soc Am, vol. 64, 1978, pp 1386-1391

[6] R. Probst and B. L. Lonsbury-Martin and G. K. Martin,"A review of otoacoustic emissions", J Acoust Soc Am, vol. 89 nr.5, 1991, pp 2027-2067

[7] V. M. Eguiluz and M. Ospeck and Y. Choe and A. J. Hudspeth and M. O. Magnasco,"Essential nonlinearities in hearing", Phys Rev Lett, vol.84 nr. 22, 2000, pp 5232-5235

[8] G. A. Tavartkiladze and G. I. Frolenkov and A. V. Kruglov and S. V. Artamasov, "Ipsilateral suppression effects on transient evoked otoacoustic emission", Br J Audiol, vol. 28 nr. 4-5, 1994, pp 193-204

[9] J. M. Harte, S. J. Elliot, S. Kapadia, M. E. Lutman, ”Dynamic nonlinear cochlear model predictions of click-evoked otoacoustic emission suppression”, Hearing Research, vol. 207, 2005, pp 99-109

[10] S. Kapadia and M. E. Lutman, "Nonlinear temporal interactions in click-evoked otoacoustic emissions. I. Assumed model and polaritysymmetry.", Hearing Research, vol. 146 nr.1-2, 2000 , pp 89-100

[11] S. Verhulst, "The role of temporal synchrony for the generation of otoacoustic emissions", Master Thesis, Ørsted-DTU, Technical University of Denmark, 2006. 